\title{
Optimal Waiting Position of a Home Robot for Risk Communication Considering Behavior Patterns of an Occupant
}

\author{
Akira Mita ${ }^{1, a, *}$, Keita Watanabe ${ }^{1, b}$ and Ami Ogawa ${ }^{1, c}$ \\ ${ }^{1}$ Department of System Design Engineering, Keio University, 3-14-1 Hiyoshi, Kohoku, \\ Yokohama 223-8522, Japan \\ amita@keio.jp, bw-keita@keio.jp, cami_ogawa@keio.jp
}

Keywords: Home Robot, Personal Space, Walking Pattern, Risk Communication

\begin{abstract}
The risk communication in a home between a home robot and an occupant must be smooth in a way that the home robot does not disturb the occupant lives. In this paper, we propose a new method to determine the optimal waiting position considering the personal space and the obstacles such as furniture and the occupant's walking patterns. It is shown that the distance to the wall from the occupant in the direction of the home robot and the standing or sitting posture affect most on the personal space. Furthermore, this personal space is dependent on each individual preference. The performance of the proposed method is much more feasible compared with those obtained in our previous approach.
\end{abstract}

\section{Introduction}

We observe a significant increase in the number of single person households in all generations. Our research group has proposed a new type of living system, called the "Biofied Building," in which a home robot is implemented to support the physical and mental health of an occupant. It is one of the important roles for this "Biofied Building" that the danger or the risk of environmental health including the structural health has to be communicated with the occupant in an appropriate manner. The optimal waiting position of the home robot is one of the key factors to be implemented into the system.

The robot's activities including this risk communication must be conducted in a way that the robot does not disturb the occupant lives. In our previous studies ([1], [2]), the following simple two rules have been considered to determine the robot waiting position:

1) Keep the appropriate distance from obstacles and the occupant

2) Choose areas where the occupant walking frequency is low

However, the optimal waiting positions often cannot be determined because the whole walking history should be reflected in the potential field to determine the waiting positions. Furthermore, the appropriate distance from an occupant is typically determined on a trial and error basis and the effects of individual and environmental differences are not considered.

A method to determine the optimal position and distance is determined in this paper for smooth communication between an occupant and a home robot considering personal space and environmental factors. Furthermore, we propose a new method to use a repulsive potential field based on the probability of choosing the next walking path, which is predicted based on the occupant's current position, instead of using the occupant's whole walking history. We first extracted the factors that affect the personal space for communication based on experiments. As a result, we reveal that the distance to the wall behind the robot and the occupant posture 
(standing/sitting) affect the interaction distance. Thus, the personal space descriptions can be generalized using these two factors.

A series of experiments were conducted to show the validity and versatility of the proposed method. The proposed method could reliably estimate appropriate waiting positions of a home robot while avoiding collision.

\section{Proposed method}

The optimal waiting position of a home robot is determined considering the following two areas.

1) The area where the occupant can comfortably communicate with the home robot. This area is determined considering a personal space that is defined for each individual and walking paths. This favorable area is modeled by an attractive potential. The potential values are dependent on the probability of the occupant walking patterns.

2) The area where the robot is not able to access due to the obstacles such as furniture and the occupant, and the area where the access of the robot is not allowed. The prohibited areas include bathrooms and closets. This inaccessibility is modeled by a repulsive potential. The potential values are dependent on the distance to the obstacles.

We employed the personal space defined by the model proposed in the reference [3]. The size of this area is dependent on the individual preferences, the environments and the other conditions. This space is thus person-dependent. When the personal space is determined, the attractive area is defined by the area close to the boundary of the personal space. This attractive area is assumed to be only available in front of the occupant as described by a blue colored area in Fig. 1. In the figure, the occupant is depicted by a simple symbol described in Fig. 2.

To calculate the repulsive potential, we need to know the position of furniture, the area where robot is not able to approach and the occupant walking patterns. The position of furniture and the area will be pre-assigned. Thus, the important process for us is to predict the occupant walking patterns. We employed a random walk model to represent the walking patterns that allows the occupant move into forward, right, backward and left with a certain probability. In our simulation, we assume that the probability to move to the destination at each step is $60 \%, 65 \%$, or $70 \%$. A simulation example is shown in Fig. 3.

The flowchart of estimating the optimal waiting position for a home robot is presented in Fig. 4. The lower the potential field, the more suitable the home robot will be. The total potential is represented by the repulsive potential minus the attractive potential.

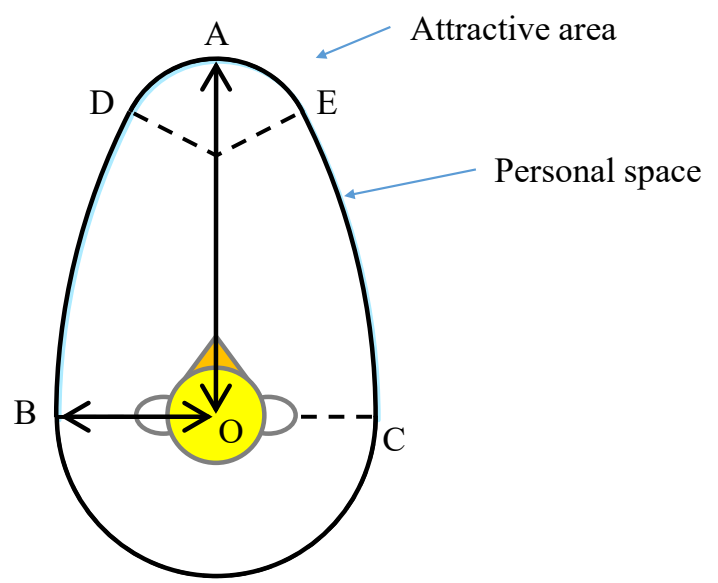

Figure 1 Definition of personal space ${ }^{[3]}$ and attractive area 


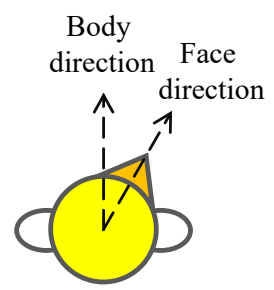

Figure 2 An occupant illustration showing the body and face directions
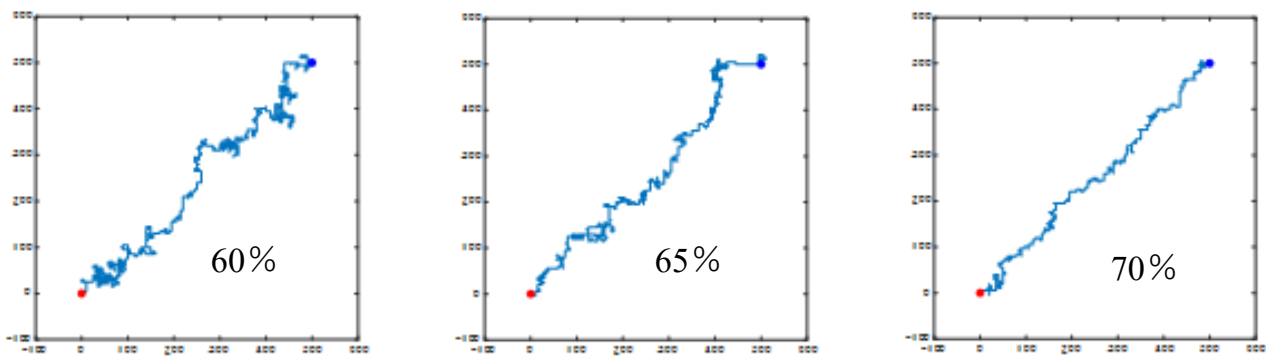

Figure 3 Simulated occupant walk using random walk with higher weights to the destination

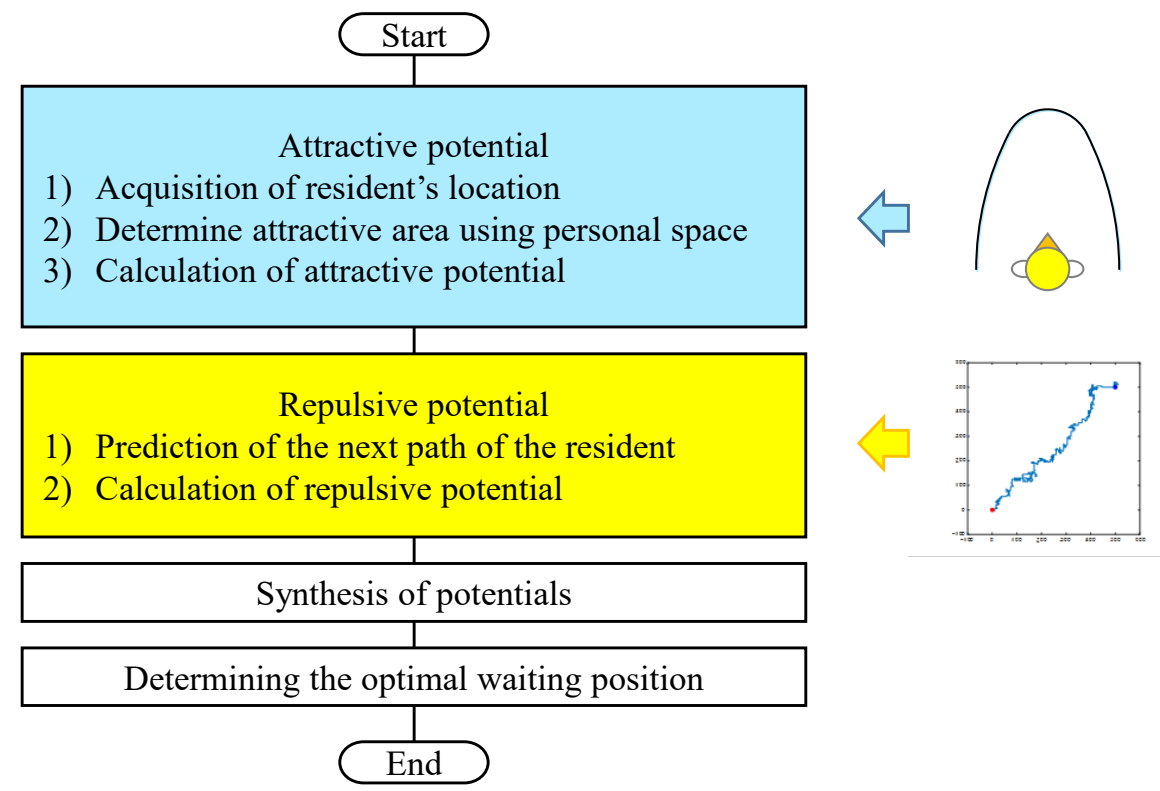

Figure 4 Flowchart of determining optimal waiting position for a home robot

\section{Experimental evaluation of personal space and attractive area}

The size of the personal space is dependent on several parameters. To identify the key parameters for our home robot, a series of experiments were conducted. The specifications of our prototype home robot are depicted in Table 1. The photo of the robot is shown in Fig. 5. The 
experimental setup is presented in Fig. 6. The parameters considered in the experiments are posture, sex, height of the subjects. In addition, among a subject's life patterns, if he or she was living with a pet or not was also considered. For a home robot, approach direction of front and side was considered. The distance between the occupant and the wall was also considered as shown in Fig. 6.

The total number of the subjects is 19, 13 males and 6 females of low twenties. It turned out that the most dominant parameters are posture (sit or standing) and the distance to the wall. The width of the attractive area around the personal space was set $+30 \mathrm{~cm}$ and $-30 \mathrm{~cm}$ determined from the standard deviation as represented by the blue area in Fig. 1.

Table 1 Specification of the robot used for experiments

\begin{tabular}{|c|c|}
\hline Items & Values \\
\hline Size $(\mathrm{mm})$ & $396(\mathrm{~W}) \times 320(\mathrm{D}) \times 540(\mathrm{H})$ \\
\hline Wheel diameter $(\mathrm{mm})$ & 150 \\
\hline Maximum speed $(\mathrm{ms})$ & 5.0 \\
\hline Micro-controller & Mbed LPC1768 \\
\hline Longest running time (hours) & 3.5 \\
\hline Motor-wheel connection & Pulleys and belts \\
\hline
\end{tabular}

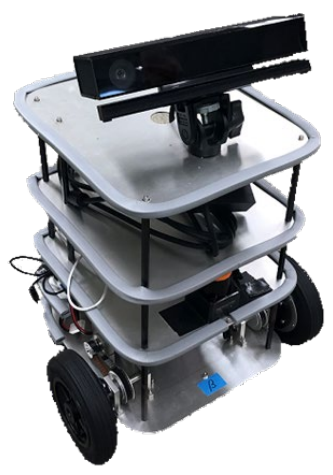

Figure 5 Prototype home robot e-bio $T \beta v 2$

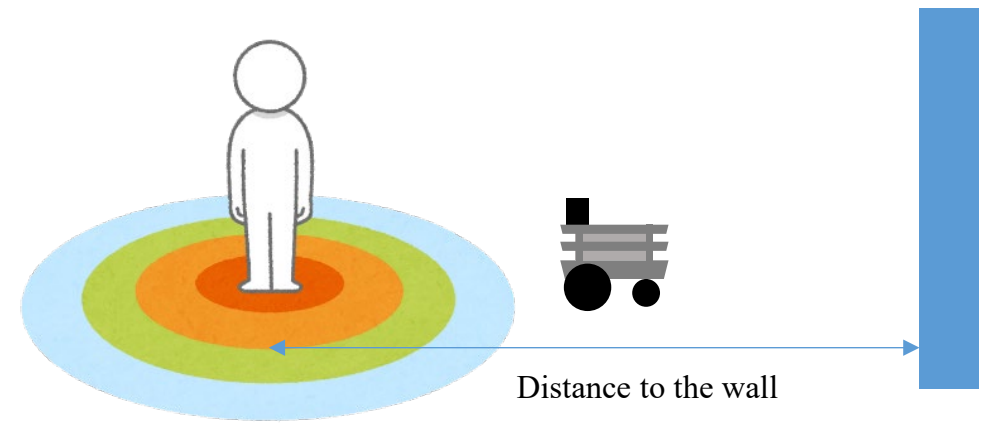

Figure 6 Experimental setup for evaluating factors affecting personal distance 


\section{Simulation of optimal waiting positions}

A simulation was conducted for the room depicted by Fig. 7. Kitchen, bath rooms and walk-in closet are the areas where the home robot can not approach. This room is chosen from the database CASAS (Center for Advanced Studies in Adaptive Systems) ${ }^{[4],[5]}$ in which the life patterns for the duration of two months are recorded. Using the recoded patterns, the probability of the next walking direction was predicted using a Markov model. To consider the variation, the random walk depicted by Fig. 3 were employed.

In Fig. 8, an example is shown. The left result is based on the conventional method[4] where all the walking patterns are included to determine the repulsive potential and no attractive potential is considered. The right result is based on the proposed method. Two red areas indicate the optimal waiting positions suggested by the current method. It is clearly shown that the wider and more appropriate waiting area is available using the proposed method.

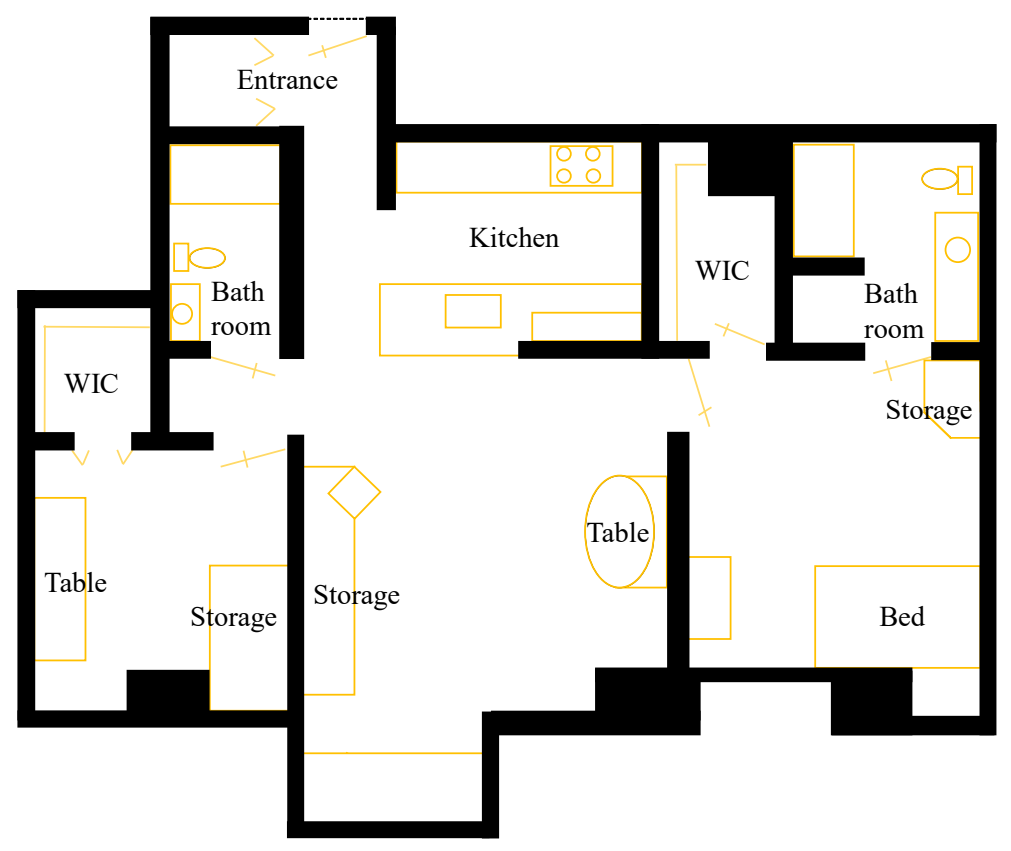

Figure 7 Floor plan of the house used for simulation
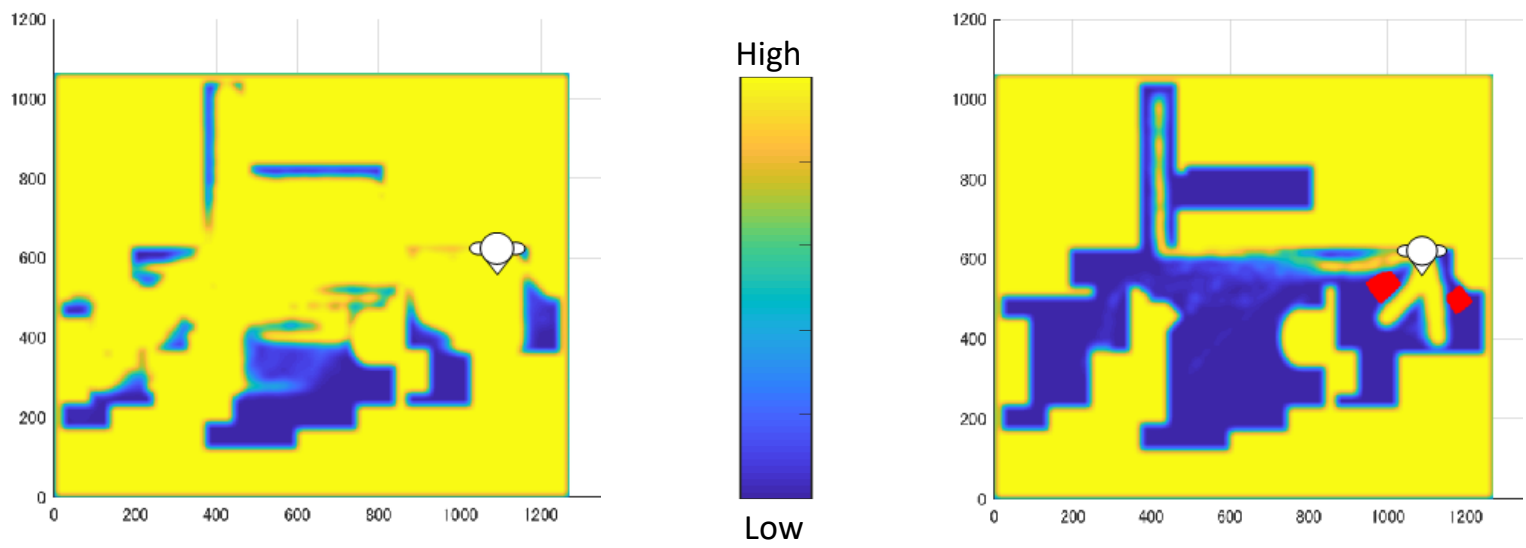

Figure 8 Potential map using a conventional method (left) and the proposed method (right) 


\section{Concluding remarks}

A significant increase is observed in the number of single person households in all generations in Japan. We have been working on preventing the danger or the risk of environmental health including the structural health has to be communicated with the occupant in an appropriate manner. This research is aimed at planning the optimal waiting position of the home robot for communicating with the occupant.

The optimal waiting position was determined in such a way that the robot will be in the favorable area with respect to the occupant's personal space and does not interfere with the obstacles. The furniture and the walking paths of the occupant are the most important obstacles to be considered. In our previous studies, we only considered the obstacles and did not include the personal space to decide the optimal position. In addition, the occupant's walking patterns were described by a probability distribution using all walking histories. Thus, it took a lot of time and data to decide the positions. In this study, the prediction of walking path is implemented using a Markov model based on the available walking patterns recorded by the time of prediction. In this paper, simulations were based on the data provided by the CASAS database. It was shown that the performance of the proposed method was much more feasible and flexible than those obtained using our previous approach.

\section{Acknowledgements}

This research was partially supported by a grant from the Japan Society for the Promotion of Science (JSPS KAKENHI 18H00968).

\section{References}

[1] Iida, M., Mita, A.: A Study on Chasing Algorithm and Walking Parameters Acquisition using Home Robot, AIJ Journal of Technology and Design, Vol. 22, No. 50, pp. 253-257, 2016.2 (in Japanese). https://doi.org/10.3130/aijt.22.253

[2] Kimura, T., Ogawa, A., Mita, A.: Path Planning in Residential Space of Home Robot Incorporating Waiting Behavior, AIJ Journal of Technology and Design, Vol. 26, No. 62, pp. 407-411, 2020.2 (in Japanese). https://doi.org/10.3130/aijt.26.407

[3] Liu, C.H., Ooeda, Y., Sumi, T.: A Model for Pedestrian Movement with Obstacle Evasion using Personal Space Concept, Journal of Japan Society of Civil Engineers D, Vol. 64, No. 4, pp. 513-524, 2008 (in Japanese). https://doi.org/10.2208/jscejd.64.513

[4] Cook, D.J., Crandall, A.S., Thomas, B.L. and Krishnan, N.C.: CASAS: A smart home in a box. Computer, Vol. 46, No. 7, pp. 62-69, 2012. https://doi.org/10.1109/MC.2012.328

[5] Washington State University, CASAS: Center of Advanced Studies in Adaptive System. http://casas.wsu.edu/datasets/ (accessed 07/08/2020). 